\title{
Baccharis latifolia: UNA ASTERACEAE POCO VALORADA CON POTENCIALIDAD QUIIMICA Y MEDICINAL EN EL NEOTRÓPICO
}

Fecha de recepción: 05 de enero de 2016 • Fecha de Evaluación: 01 de marzo 2016 • Fecha de aceptación: 16 de marzo de 2016

\section{Baccharis latifolia: A LOWLY-VALUED ASTERACEOUS PLANT WITH CHEMICAL AND MEDICINAL POTENTIAL IN NEOTROPICS}

Jessica Prada1', Luisa Orduz-Diaz¹, Ericsson Coy-Barrera ${ }^{1 *}$

RESUMEN

Baccharis es un género de plantas muy numerosa (400-500 especies) altamente distribuidas en el neotrópico, dentro de las que se encuentra B. latifolia (Ruiz \& Pav.) Pers. Esta planta presenta diversos usos en medicina tradicional en América Latina, dentro de los cuales se destacan usos analgésicos y antiinflamatorios, los cuales ha sido objeto de estudios adicionales que se han encauzado en su validación. Sin embargo, no hay una conexión importante entre las propiedades medicinales o farmacológicas con la composición química, salvo aquellos casos donde se justifica la actividad con otros estudios donde se aíslan compuestos que poseen actividad. Por tanto, el escenario indica que, pese a que existe información importante, los datos son marginales o separados entre sí, lo que permite justificar que es todavía importante realizar estudios adicionales con fines quimioprospectivos. Por consiguiente, en el presente trabajo se describe, de forma generalizada, la información reportada a la fecha en estudios químicos y biológicos de la familia Asteraceae, del género Baccharis y de la especie B. latifolia, la cual, pese a tener un potencial claro en el neotrópico, es una planta poco conocida y valorada que merece mucha atención.

Palabras Clave: Asteraceae, Baccharis, Baccharis latifolia, flavonoides, diterpenos.

1 Laboratorio de Química Bioorgánica, Facultad de Ciencias Básicas y Aplicadas, Universidad Militar Nueva Granada. *Autor corresponsal. E-mail: inquibio@unimilitar.edu.co 


\section{ABSTRACT}

Baccharis is a genus of very large plants (400-500 species) highly distributed in the Neotropics, within B. latifolia (Ruiz \& Pav.) Pers is found. This plant in Latin America has many uses in traditional medicine such as painkillers and anti-inflammatory, which has been the aim of additional studies for validation purposes. However, there is not an important connection between medicinal or pharmacological properties and chemical composition, except in those cases where the activity is justified with other studies which bioactive compounds are isolated. Therefore, the scenario indicates that although there is important information, the data is marginal or separated from each other, justifying further studies towards its chemoprospecting. Consequently, in this paper is described, in general terms, the information reported to date in chemical and biological studies on the Asteraceae family, Baccharis genus and B. latifolia. Despite this plant has a clear potential in the neotropics, it is a little known and valued plant that deserves a lot of attention.

Keywords: Asteraceae, Baccharis, Baccharis latifolia, flavonoids, diterpenes.

\section{NTRODUCCIÓN: LA FAMILIA ASTERACEAE}

La familia de las compuestas (Asteraceae) constituye el grupo vegetal más diverso de plantas vasculares sobre el planeta (Bremer, 1994; Smith et al., 2004). Se estima su riqueza en aproximadamente 25.000 especies y más de 1.500 géneros. Su distribución se da por todo el mundo, excepto en la Antártida (Cabrera et al., 2000; Funk et al., 2009). Son abundantes en regiones semiáridas, tropicales y subtropicales, Provincia del Cabo, Sur de América, Australia y Región Mediterránea, Rusia y Estados Unidos (Oliva et al, 2003). Debido a su alta capacidad de adaptación y supervivencia son muy biodiversas, con la posibilidad de crecer en zonas alpinas de altas montañas hasta plantas con ciclo CAM (Crassulacean Acid Metabolism), que crecen en zonas áridas/desérticas (e.g., géneros Senecio y kleinia) (Funk et al., 2009; Oliva et al, 2003).

Entre los géneros más importantes de la familia Asteraceae se encuentran el Senecio (1.250), Vermonia (1.000), Cousinia (650), Eupatorium (600),
Centaurea (600), Artemisia (550), Baccharis (500) (Verdi et al., 2005). Se distinguen algunas especies ornamentales de gran importancia económica como el Girasol (Helianthus annuus) (Wiersema y Leon, 2013) y géneros como Dendranthema, Argytanthemum, Leucanthemum, Dahlia, Tagetes, Senecio, así como otras especies conocidas como comestibles tales como Cichorium (escarola), Cynara (alcachofa), Helianthus (girasol), Taraxacum (diente de león), y Lactuca (lechuga). Además, otras plantas como las especies de los géneros Tanacetum y Pulicaria tienen propiedades insecticidas, y otro gran número de plantas con propiedades medicinales, entre las que se cuenta la manzanilla (Matricaria chamomilla), la mercadela (Canendula officinalis), la artemisa (Artemisia vulgaris), entre otras (Tapia, 2010). Además esta familia es particularmente rica en sesquiterpenos, un grupo de metabolitos secundarios que poseen propiedades biológicas y farmacológicas (Cabrera et al., 2000; Oliva et al, 2003), 
entre los que se destacan aquellos sesquiterpenos que han presentado actividad antitumoral (Daniel, 2006). De hecho, el premio Nobel en medicina del año 2015 fue otorgado en parte a la Prof. Youyou Tu, por el descubrimiento y desarrollo de la artemisinina, una sesquiterpenlactona con propiedades muy efectivas contra el parásito causante de la malaria, que fue aislado de una Asteraceae, la Artemisia annua, una planta muy utilizada en la medicina tradicional de China (Callaway y Cyranoski, 2015).

Colombia no es la excepción y por ende la familia Asteraceae está representada en todos los climas. Sus especies exhiben una amplia plasticidad estructural y un notable ajuste ecológico; hecho que permite encontrar algunas especies en desiertos, otras en paramos o en manglares, así como en las cordilleras o en las sabanas e incluso en la selva amazónica (Díaz-Piedrahita, 1989). El Occidente de la Sabana de Bogotá (municipios de Subachoque, El Rosal, y Madrid) es una de la más diversas con 59 géneros/155 especies (Díaz-Piedrahita y Rodríguez-Cabeza, 2012).

\section{EL GÉNERO Baccharis}

El género Baccharis es uno de los más ricos en especies dentro de la familia Asteraceae. Está compuesto por más de 400 especies (Guiliano, 2001; Pires \& Becker, 2010; Verdi et al., 2005), distribuidas principalmente en regiones tropicales del continente (Troiani, 1985) como: Brasil, Argentina, Colombia, Chile y México (Pires \& Becker, 2010; Verdi et al., 2005). Su distribución es exclusivamente americana, va desde el Sur de los Estados Unidos hasta el extremo austral de Chile y Argentina (Giuliano, 2001). Su alta concentración de especies en Brasil y los Andes indica que una de estas áreas es probablemente el centro de origen pero este hecho todavía no es claro (Verdi et al., 2005), dado que se pueden encontrar ampliamente diversificado en gran variedad de ambientes (Giuliano, 2001). A pesar de todo esto, este género no ha sido monográfiado y, por lo tanto, no se tiene el número correcto de especies que lo conforman. Sin embargo, Malagarriga, en 1976, pese a la fecha lejana, fue quien a ese momento realizó uno de los mayores aportes al incluir la mayor cantidad de nombres válidos y sinónimos publicados hasta ese día, donde se reconoció un total de 431 especies y 80 entidades intraespecíficas. A estas cifras se debe agregar 37 especies y 7 taxones intraespecíficos provenientes y descritos a través de nuevas combinaciones; también cerca de 40 nombres que tampoco fueron mencionados por el autor (cfr. Index Kewensis y Gray Herbarium Card Index) (Guillano, 2001).

La sistemática del género, básicamente neotropical, es muy confusa, debido a la gran cantidad de especies publicadas y sus distintas y variadas formas, además de la falta de estudios formalmente rigurosos (Cuatrecasas 1968). Las especies del género Baccharis generalmente son arbustos, que miden en promedio de 0.5 a $4.0 \mathrm{~m}$ de altura (Verdi et al., 2005), aunque se extiende también a plantas perennes herbáceas y subarbustos (Jasinski et al., 2014). No obstante, la mayoría de las plantas de Baccharis se pueden distinguir por su hoja o tallo alado (Freire et al., 2007), siendo las hojas morfológicamente diferentes, lo que ayuda bastante a la identificación (Jasinski et al., 2014). Además, todas las hojas de las especies del género se caracterizan por ser pubescentes (solo unas pocas como B. dracunculifolia y $B$. trinervis son subglabro en la madurez). Freire (2007) encontró que dos grupos de indumento caracterizan las plantas medicinales: el primero con tricomas aislados y el segundo con tricomas en mechones (vellosidad uniseriada con células terminales ramificadas).

\section{USOS MEDICINALES DE GÉNEROS DE Baccharis}

Verdi et al (2005) reportaron que, además de otros usos igualmente significativos, las especies 
de Baccharis tienen gran importancia socioeconómica en los estados de Santa Catarina, Paraná, São Paulo y Rio Grande del Sur, entre otras regiones de Brasil, porque es usada en la medicina tradicional para el control y tratamiento de diferentes enfermedades. La consumen principalmente cómo té, para aliviar dolencias de estómago, el hígado, inflamaciones, anemia, diabetes, desintoxicación del cuerpo y también para enfermedades de la próstata (Korbes, 1995; Correa, 1984). En otros lugares del continente, diversas especies de este género son también utilizadas tradicionalmente para el tratamiento de varias dolencias, tales como contusiones, inflamaciones, también como antimicrobiano y desinfectante (Martínez et al., 2010). A manera de compilación, en la Tabla 1 se muestran los diversos usos que se le han dado tradicionalmente a varias especies del género Baccharis, lo cual indica la enorme potencialidad que las plantas de este género poseen y que permiten poner de manifiesto un escenario claro para futuros estudios que lleven, por un lado, a la validación de tales usos, y, por el otro, a proporcionar, dentro de estudios adicionales de bioprospección, un valor agregado a estas plantas tan versátiles.

\section{Fitoquímica del género Baccharis}

Las propiedades que han demostrado especies del género Baccharis se deben por supuesto a su composición química, basada principalmente en flavonoides, diterpenos y triterpenos (Verdi et al., 2005), donde los flavonoides se distinguen por conferir protección/resistencia frente al ataque de microorganismos (Martínez et al., 2010). También se han obtenido, aunque en menor proporción, cumarinas y aceites esenciales (Abad y Bermejo, 2007). A pesar de todo lo anterior, hasta el momento no se ha establecido un marcador químico para caracterizar a las especies de Baccharis (Lonni et al., 2003; Lonni et al., 2005). Dado este contexto y panorama, el género Baccharis ha sido objeto de una variedad de estudios fitoquímicos y de actividad biológica, iniciando su estudio a principios de 1900 y, hoy en día, hay más de 150 compuestos aislados e identificados de este género (Abad y Bermejo, 2007). A continuación se describen los núcleos más representativos de metabolitos aislados de especies del género Baccharis con algunos ejemplos estructurales más particulares y distintivos.

\section{Flavonoides}

Los flavonoides son compuestos producidos casi universalmente en las plantas. Algunos de ellos son los responsables de la coloración de las flores, frutos y a veces de las hojas (Havsteen, 2002). Estos compuestos corresponden a un grupo de metabolitos secundarios que se encuentran formados por un anillo aromático unido por lo menos a un grupo oxhidrilo (Bedascarrasbure et al., 2004). Por lo general están presentes como agliconas libres y otras veces como glicósidos, lo cual correspondería a una característica de flavonoides de la familia Asteraceae. Se tiene un registro de 298 flavonoides en Baccharis con 109 compuestos diferentes, de los cuales 24 unidades son de flavanona (2) y 85 unidades de flavona (1), en el que el $48 \%$ posee un oxígeno en C3. No obstante, el patrón de oxigenación puede ocurrir también en C5 y C7, del anillo $A, y$ C4', del anillo B (Verdi et al., 2005).

A manera de ejemplos, en estudios fitoquímicos realizados con el extracto en metanol de $B$. pseudotenuifolia se aislaron los flavonoides hispidulina (3), naringenina (4), 3'-metoxiluteolina (5), apigenina (6), kaempferol (7), eriodictiol (8), quercetina (9). Los flavonoides (3) y (4) también fueron aislados de B. ligustrina (Moreira et al., 2003) y, en las especies B. gaudichaudiana y B. rufescens se han aislado dos flavonoides en común: cirsimaritin (10) y cirsiliol (11). Así mismo, las especies de Baccharis también presentan reportes para los casos de 


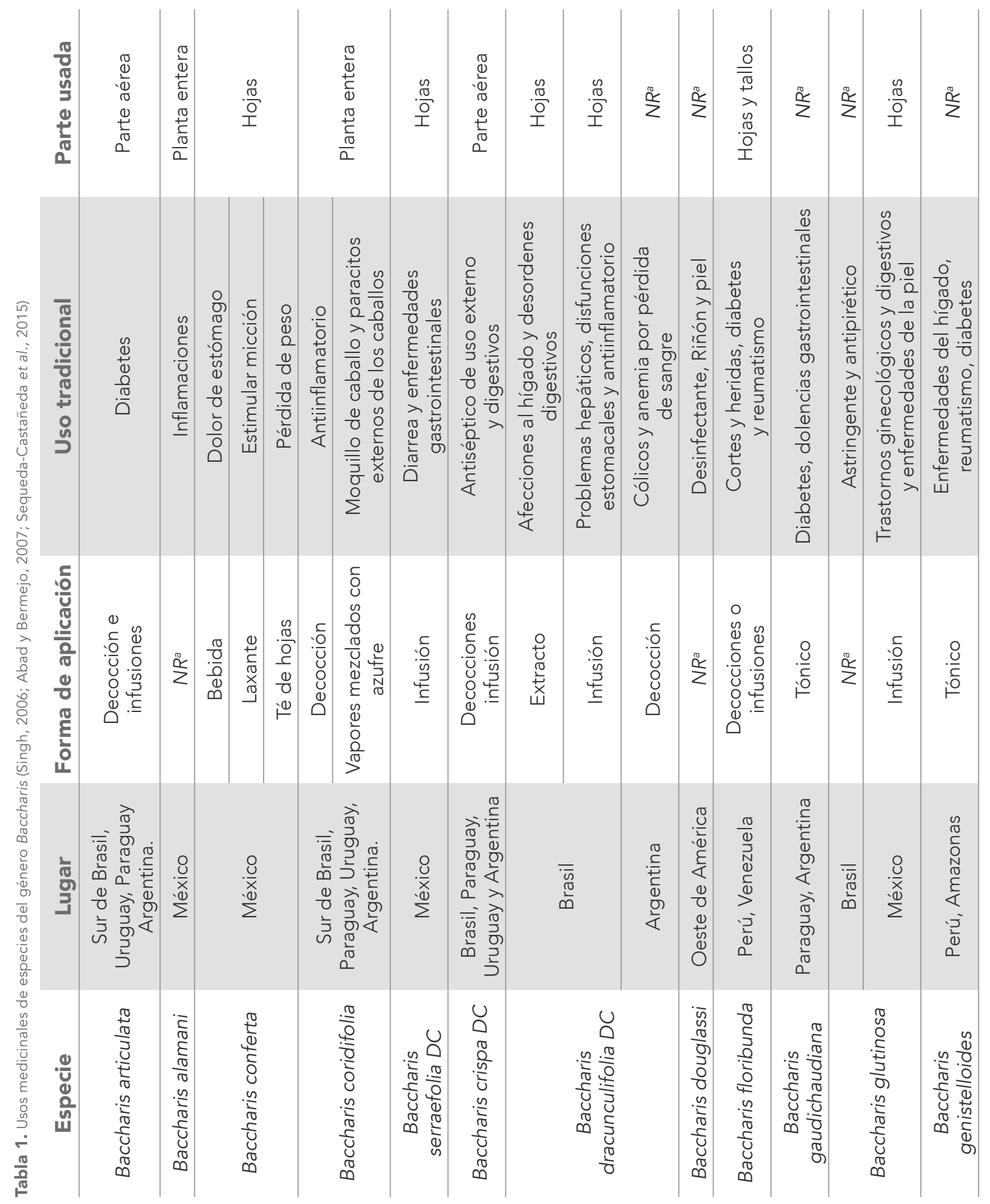




\begin{tabular}{|c|c|c|c|c|c|c|c|c|c|c|c|c|c|c|}
\hline 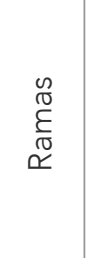 & $\begin{array}{l}\frac{0}{0} \\
\frac{.0}{1} \\
\end{array}$ & 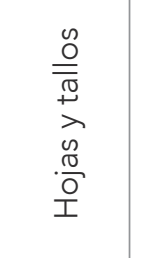 & 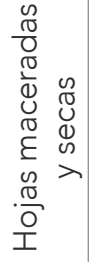 & 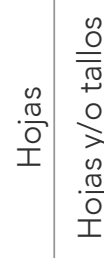 & 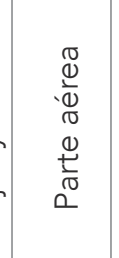 & $\begin{array}{l}\frac{0}{0} \\
\frac{10}{ \pm 0} \\
\frac{0}{2} \\
\frac{.0}{0} \\
\frac{0}{1}\end{array}$ & $\begin{array}{l}\frac{0}{0} \\
\frac{0}{1} \\
\frac{1}{1}\end{array}$ & $\begin{array}{l}\pi \\
0 \\
0 \\
0 \\
0 \\
0 \\
0 \\
\frac{ \pm}{\sigma} \\
0\end{array}$ & & $\begin{array}{l}\stackrel{0}{0} \\
\stackrel{0}{0} \\
\stackrel{0}{\lambda} \\
00 \\
\frac{00}{0} \\
\frac{0}{1}\end{array}$ & 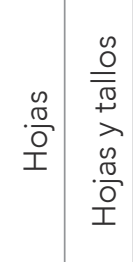 & 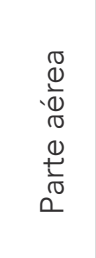 & 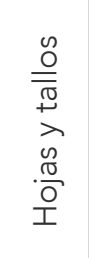 & $\begin{array}{l}\frac{\pi}{2} \\
\frac{\pi}{0} \\
\frac{\pi}{0} \\
\frac{\pi}{\sigma} \\
\frac{\pi}{0} \\
0 \\
\end{array}$ \\
\hline 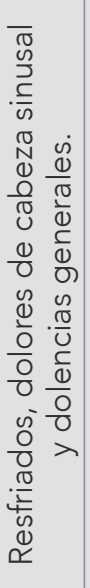 & $\begin{array}{l}\stackrel{y}{ \pm} \\
\stackrel{\pi}{0} \\
\stackrel{0}{0} \\
\sim\end{array}$ & 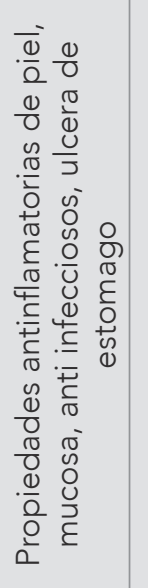 & 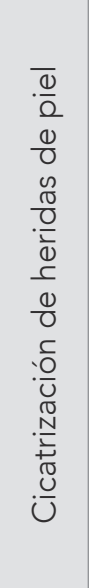 & $\begin{array}{l}\frac{\pi}{\pi} \\
\frac{\pi}{0} \\
\frac{0}{1}\end{array}$ & 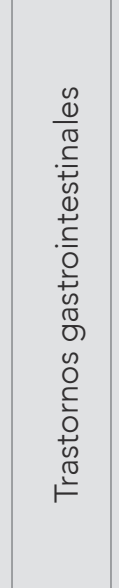 & 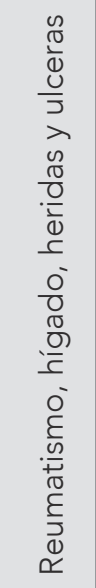 & 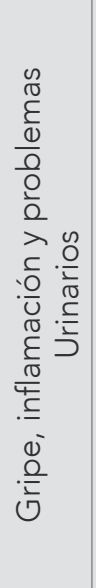 & 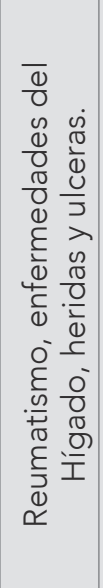 & 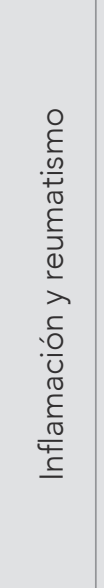 & 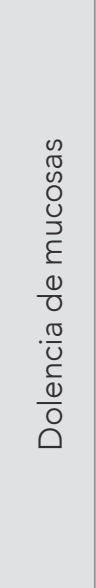 & 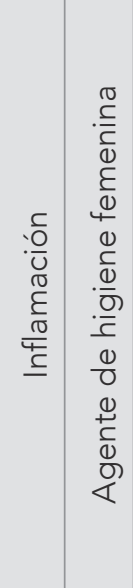 & 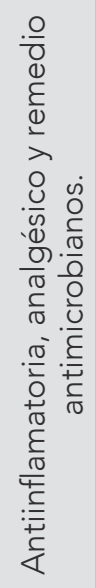 & 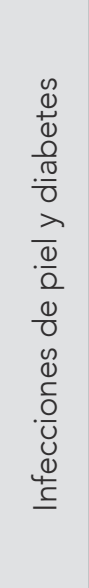 & 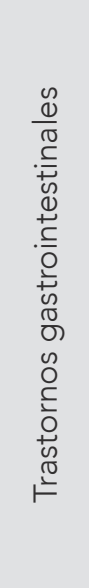 \\
\hline 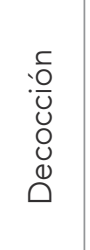 & $\frac{\mathrm{o}}{2}$ & $\stackrel{\oplus}{\oplus}$ & $\frac{0}{\frac{0}{0}}$ & 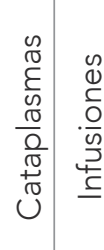 & 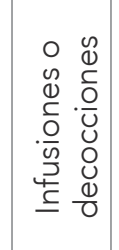 & 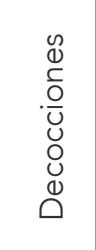 & 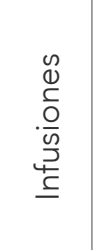 & 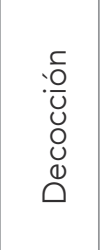 & $\frac{a}{2}$ & 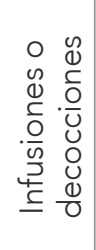 & 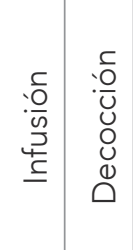 & 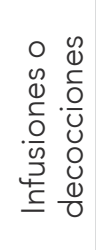 & 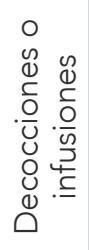 & 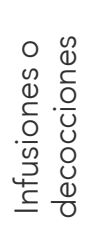 \\
\hline 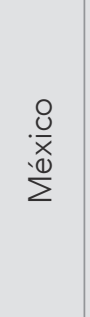 & $\begin{array}{l}\overline{\bar{\pi}} \\
\overline{0} \\
\bar{\nu}\end{array}$ & $\begin{array}{l}\overline{\bar{g}} \\
\overline{\tilde{D}} \\
\bar{n}\end{array}$ & & 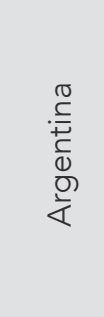 & $\begin{array}{l}\stackrel{0}{x} \\
\frac{.}{x} \\
\sum\end{array}$ & 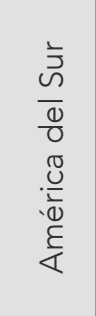 & $\begin{array}{l}\stackrel{0}{x} \\
\frac{.}{x} \\
\sum\end{array}$ & $\begin{array}{l}\frac{.0}{0} \\
\frac{\hat{\varepsilon}}{0} \\
\end{array}$ & $\frac{\stackrel{0}{\stackrel{0}{Z}}}{\bar{D}}$ & 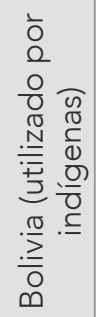 & 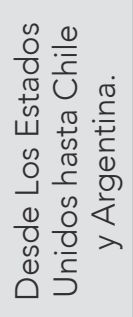 & $\begin{array}{l}\bar{o} \\
\frac{0}{0} \\
\frac{0}{3} \\
\bar{u}\end{array}$ & $\begin{array}{l}\frac{0}{0} \\
\frac{\pi}{N} \\
\frac{0}{0} \\
\frac{D}{0}\end{array}$ & $\begin{array}{l}\stackrel{0}{\cdot} \\
\stackrel{\cdot}{x} \\
\sum\end{array}$ \\
\hline 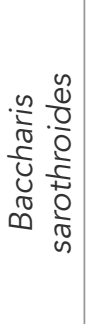 & 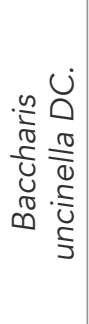 & 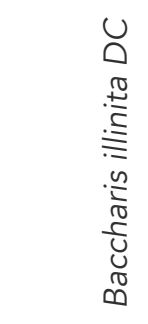 & & 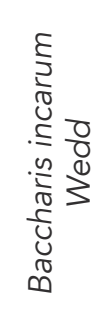 & 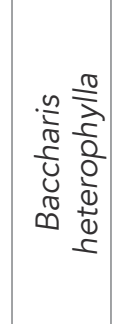 & 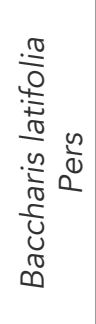 & 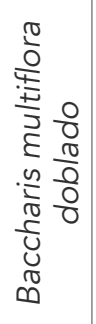 & 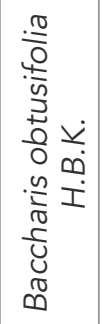 & 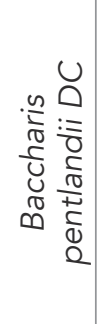 & 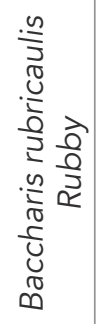 & 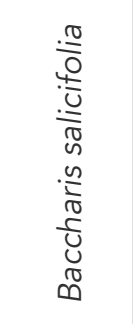 & 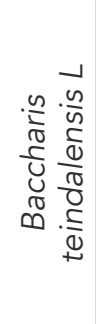 & 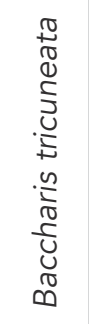 & 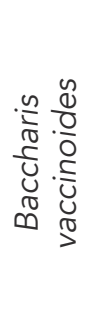 \\
\hline
\end{tabular}


dolencias hepáticas, como es el caso de B. trimera, cuyo extracto crudo antihepatotóxico mostró la presencia de quercetina (9), nepetina (12), apigenina (6), hispidulina (3), entre otros, concluyendo que tal eficacia del extracto bruto podría explicarse en gran parte por la presencia de los compuestos de núcleo flavonoide identificados (Soicke y LengPeschlow, 1987). Figura 1.
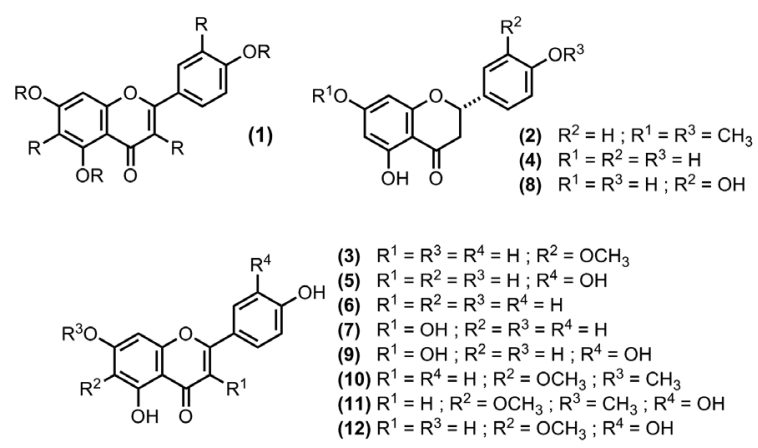

Figura 1. Flavonoides aislados de especies del género Baccharis.

Los flavonoides también han sido estudiados durante décadas por su amplia gama de actividades biológicas (Anzenbacher y Zanger, 2012). Numerosos estudios han asociado a los flavonoides con beneficios en la salud, basadas principalmente en importantes propiedades antioxidantes, ya que reducen la peroxidación lipídica y el efecto negativo de los radicales libres, contribuyendo a la reducción del riesgo de enfermedades cardiovasculares (Bedascarrasbure et al., 2004; Havsteen, 2002), diabetes y algunos tipos de cáncer (Andersen y Jordheim, 2006; Raymond, 2009; Tapia et al., 2004). Por su parte, Grecco et al., (2012) reportaron un flavonoide aislado de $B$. retusa, nombrado como sakuranetina, evaluado in vitro contra Leishmania spp, el cual presentó una razonable actividad antiparasitaria.

\section{Triterpenos}

Jarvis et al., (1991), en su estudio en Baccharis, demostró que este tipo de metabolitos se biosintetizan en algunas especies después de polinización de plantas femeninas, como es el caso $B$. coridifolia. Aunque existen pocos reportes sobre la composición de triterpenos en el género Baccharis, los compuestos más comunes son triterpenoides de núcleo oleanano (Abad y Bermejo 2007), como el ácido oleanólico (13) y el maniladiol (14), como es el caso de B. vaccinoides, B. sarothroides y B. heterophylla (Arriaga-Giner et al., 1986). Figura 2.

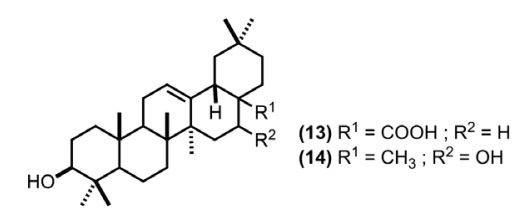

Figura 2. Triterpenos aislados de especies del género Baccharis.

\section{Diterpenos}

Los diterpenos también son metabolitos secundarios muy característicos del género Baccharis. El núcleo más común es del tipo clerodano y neoclerodano, aunque también se han aislado derivados de kaurano y labdano (Abad et al., 2005). Así, de B. gaudichaidiana se aislaron dos diterpenos de núcleo neo-clerodano, denominados gaudichaunólido B (15) y bacchariol (16) (Abad y Bermejo, 2007), y de núcleo clerodano como los ácidos hautriwaico (17) y $2 \beta$-hidroxihautriwaico (18), obtenidos de las resinas de las hojas de las especies $B$. vaccinoides, $B$. sarothroides y $B$. heterophylla (Arriaga-Giner et al., 1986). Por su parte, de la especie B. minutiflora se aisló una serie de diterpenos de tipo kaurano, dentro de los cuales se destacan el kaur-16-eno (19), ácido kaur-16-en-19-óico (20), y kaur-16-en-19ol (21) (Bohlmann et al., 1982), de la especie B. salicifolia se aislaron los diterpenos labd-13-en-8,15diol (22) y labd-13-en-8,15,16-triol (23) (Jakupovic et al., 1990), y de la parte aérea de B. pingraea se 
aisló el labd-8-en-2,3,15-triol (24) y el ácido labd8-en-2,3-dihidroxi-15-óico (25) y sus respectivos glicósidos (Zdero et al., 1990), los cuales corresponden a metabolitos de núcleo labdano. Figura 3.
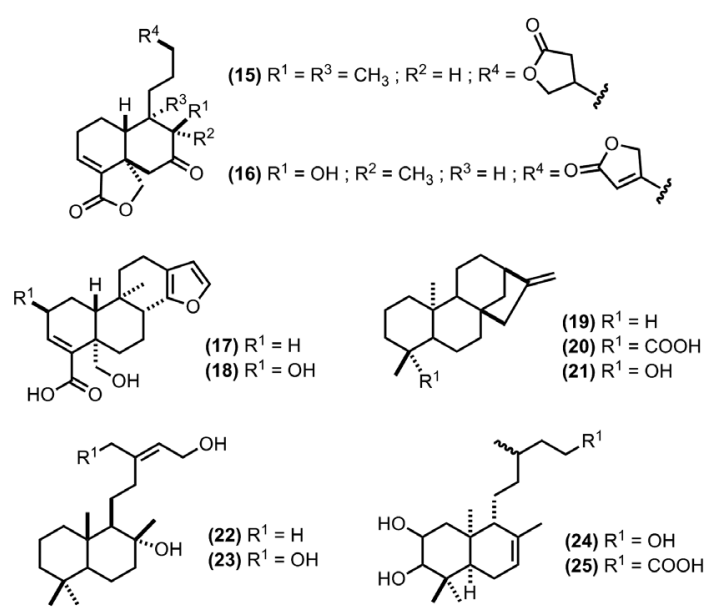

Figura 3. Estructuras de diterpenos aislados del género Baccharis.

\section{Cumarinas}

Aunque no es frecuente este tipo de compuestos en Baccharis, a partir de extractos de hojas de B. darwiniis se aislaron tres cumarinas llamadas $5^{\prime}$-hidroxiaurapteno (anisocoumarin H) (26), aurapteno (7-geraniloxicumarina) (27) y 5'-oxoaurapteno (diversinina) (28). La anisocoumarina $\mathrm{H}$ y diversinina han demostrado tener actividad antifungica (Kurdelas et al., 2010). Figura 4.

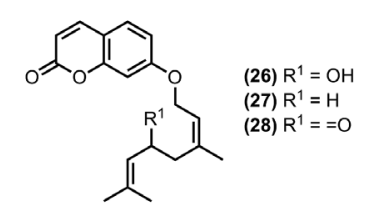

Figura 4. Estructuras de cumarinas aislados del género Baccharis.

Compuestos encontrados en aceites esenciales

Los aceites esenciales han sido obtenidos de las hojas de varias especies del género, los cuáles han servido para propósitos taxonómicos, predominando de forma constante la ocurrencia de sesquiterpenos especialmente de aquellos de la ruta del germacreno, mientras que monoterpenos son más bien dependientes de factores estacionales (Simões-Pires et al., 2005; Lago et al., 2008). Así, a partir de B. racemosa, B. linearis, y B. obovata se han identificado compuestos como limoneno (29), $\delta$-cadineno (30), mirceno (31) (Molares et al., 2009); de B. salicifolia compuestos como $\alpha$-tujeno (32), $\alpha$-y $\beta$-pineno (33), $\alpha$ -felandreno (34), limoneno, $\beta$-ocimeno (35), terpinen4-ol (36) (Malizia et al., 2005a; Malizia et al., 2005b) y, por último, de la especie $B$. tenelia se obtuvo el sesquiterpeno llamado espatulenol (37) (Biurrun et al., 2005) y de la especie $B$. salicina se obtuvo el bacchariólido A (38) y B (39) (Parodi y Fischer, 1988). Figura 5

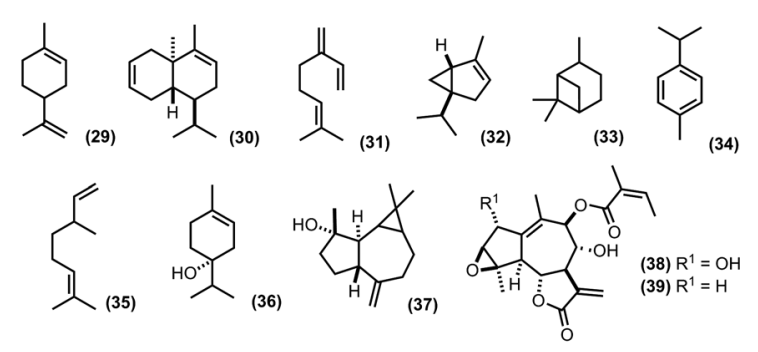

Figura 5. Compuestos identificados en aceites esenciales y algunos sesquiterpenos obtenidos de especies del género Baccharis.

\section{La Especie: Baccharis latifolia (Ruiz \& Pav.) Pers}

Esta especie se distribuye por toda la zona tropiandina, de Venezuela a Bolivia y norte de Argentina. Presenta un rango altitudinal entre los 1600 y 3800 m.s.n.m, pero predominan entre los 2500-3000 m.s.n.m. Sin embargo, tiene una amplia distribución, pues puede llegar a establecerse en un rango altitudinal bajo de hasta 1500 m.s.n.m, hasta descender de 500 a 1100 m.s.n.m. Se ha observado en zonas heladas eventualmente y con una temperatura media que oscila entre $7-19^{\circ} \mathrm{C}$ (Hoyos y Yep, 2008). Esta planta presenta una alta tolerancia a suelos pobres y difíciles, 
siendo capaz de tolerar suelos con alta pedregosidad y estaciones carentes de agua (Reynel y Leon 1990).

A nivel tradicional, B. latifolia ha sido muy usada en diferentes técnicas de cocimiento, específicamente para tratar daños e inflamaciones de las articulaciones, nervios y tendones; en infusiones para dolores de estómago, reducción de flatulencias, mejora de condiciones asmáticas, enfermedades de la matriz, cataplasma para luxaciones, torceduras, hernias, entre otras. Además, se ha registrado que esta especie presenta actividad biocida frente a los fitopatógenos Aspergillus niger y Phytophthora palmivora (Martínez et al., 2010).

\section{Descripción botánica de B. latifolia}

La especie Baccharis latifolia es un arbusto que alcanza una altura de 1,5 a 3 metros. Posee hojas simples alternas, con peciolo de 1,5 a $2 \mathrm{~cm}$ de largo, oblongo-lanceolada, de ápice acuminado, base cuneiforme, uniformemente aserrada en la margen, de color verde brillante por el haz, pegajosas y con tres nervios pronunciados que salen desde la base (Velásquez, 2007). Dado todo esto, las hojas sirven como una característica importante para diferenciarlas taxonómicamente. Las ramas son delgadas, glabras y resinosas. La inflorescencia es blanquecina paniculada, terminal y ramificada, los capítulos son numerosos dispuestos en los ápices de las ramas (Hoyos y Yep, 2008). Su involucro o umbrela es acampanado, las brácteas en tres o más hileras o series de borde hilialino (transparente). Presenta numerosas flores con corola filiforme; aquenios oblongos, vilano blancuzco de aproximadamente 1,2 $\mathrm{mm}$ de largo. Los frutos son de tipo aquenios de color café, de 4 a $5 \mathrm{~mm}$ de longitud y glabros.

\section{Usos tradicionales de B. latifolia}

Usos medicinales. Esta especie tiene importantes usos en la medicina tradicional, principalmente en los pueblos de América. Es usada en cocimiento, ya que tiene la virtud de reducir la inflamación de las articulaciones (Velásquez, 2007; Hoyos y Yep, 2008); también tiene propiedades analgésicas/antiinflamatorias útiles para adormecer nervios y tendones, facilitando de este modo el tratamiento de huesos dislocados. Se utiliza también en infusión de las hojas para el dolor de estómago causado por el frio. La cocción de hojas, tallos e inflorescencias es un tónico antidiabético y para enfermedades hepáticas. Se usan las hojas molidas en cataplasma para torceduras, luxaciones y hernias, pues son eficaces para desinflamar y fortificar las áreas afectadas. El cataplasma de hojas secas, molidas con grasa (formando una pomada) es útil para cicatrizar sin provocar infección, permitiendo el cierre de las heridas con mayor facilidad. También se usa como analgésico contra dolores reumáticos y de la cintura (Velásquez, 2007).

Uso agroforestal. Esta especie es utilizada para la protección y conservación de los suelos, ya que tiene un sistema radicular denso y corto, lo cual no genera competencia con otras plantas ni cultivos. Es útil como cercas vivas y/o cortinas rompevientos (Paredes, 2002), por su fácil propagación y tolerancia a diferentes temperaturas. También es importante para la recuperación de suelos afectados por el sobrepastoreo. Su tallo es útil para usar como leña, ya que por contener sustancias resinosas puede generar fuego fácilmente, incluso a pesar de estar fresco (Reynel y León, 1990; Brack, 1999).

Uso Artesanal. En un ámbito artesanal, las hojas de $B$. latifolia son útiles para teñir de amarillo o verde prendas u otros objetos (Paredes, 2002).

\section{Estudios químicos y de actividad biológica de B. latifolia}

A pesar de los diversos estudios hechos en otras especies de Baccharis y a los usos tradicionales de B. latifolia, sus estudios químicos podrían considerarse muy escasos, lo que llevaría a pensar que 
poco se conoce sobre la química de esta especie. De los pocos estudios realizados sobre B. latifolia se encuentra reportado principalmente compuestos terpénicos y/o esteroidales (Hoyos y Yep, 2008). Para destacar, se han reportado compuestos como $\alpha$-felandreno, canfeno (40) y óxido de cariofileno (41), aislados tanto en B. latifolia como de otras especies como B. salicifolia y B. paniculata. Con B. dranuncufolia comparte compuestos como terpinen-4-ol (42) y $\gamma$-gurjuneno (43) (Concha et al., 2014). De extracciones a partir de raíz se han aislado cuatro compuestos: uno derivado de timol (44) y tres sesquiterpenos (germacreno D (45), escualeno (46) y un sesquiterpeno sustituido altamente oxigenado (47) (Madigan et al., 1999), y de la parte aérea se aislaron siete compuestos, entre los que se cuentan diterpenos de núcleo labdano y sesquiterpenos de núcleo germacreno (Zdero et al., 1989). Figura 6

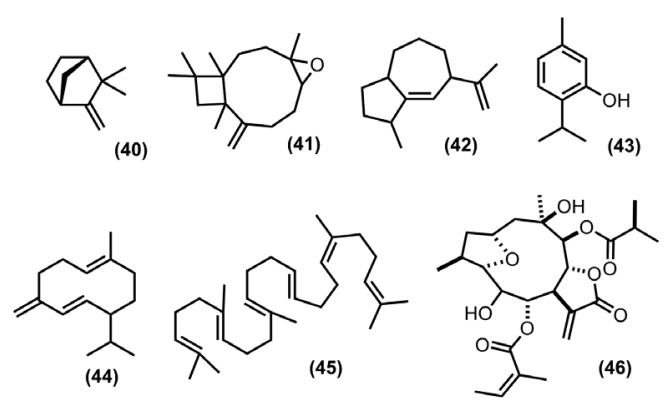

Figura 6. Estructuras de algunos compuestos aislados en B. latifolia.

No obstante, existen varios estudios reportados que se enfocan a la evaluación de la actividad biológica y/o propiedades medicinales de materiales derivados de $B$. latifolia, los cuales se describen en una compilación de datos recientemente publicada (Sequeda-Castañeda et al., 2015). Para destacar se encuentra la evaluación de la actividad antiproliferativa (Carraz et al., 2015), citotóxica y antifúngica (Zapata et al., 2010) con resultados de actividad significativos, así como dos estudios que tienen como propósito validar sus usos etnobotánicos, entre los que se cuentan la evaluación de la actividad antiinflamatoria (Abad et al., 2006) (presentando buena actividad contra COX-2 y TNF- $\alpha$ ) e inhibición en la producción de ROS y RNS (Pérez-García et al., 2001).

En conclusión, a pesar de la notable información que a la fecha existe sobre estudios enfocados a conocer la composición química y propiedades biológicas de materiales derivados de especies de Baccharis, el panorama expuesto permite apreciar que aún falta mucho por explorar en tales escenarios, dado que solo cerca de un $15 \%$ de las especies conocidas (de entre 400-500) han sido estudiadas en aspectos químicos o medicinales. Además, la química de los diterpenoides es muy variada y su potencial biológico no es la excepción; contexto que también permite indicar que es necesario plantear nuevos estudios enfocados a la bio y quimioprospección de estas especies. En este sentido, B. latifolia, aunque tiene un acervo informativo importante, los trabajos realizados se pueden considerar preliminares o demasiado separados, i.e. se ha reportado la evaluación de algunas actividades biológicas de extractos crudos de esta planta sin información química relevante o, en su defecto, se han aislado compuestos de algunas partes de B. latifolia, pero no se les conoce su potencial - propiedades biológicas por falta de la aplicación en un bioensayo. Por tanto, el reto que resulta es evidente y se basa en el hecho de plantear planes de investigación debidamente enfocados que unan tales fines, pues es claro que B. latifolia tiene potencial químico y biológico, con la ventaja de no presentar dificultades como propagación y/o consecución de biomasa, pues esta especie tiene características adaptativas y de crecimiento particulares y efectivas, que la hacen un excelente modelo para fines bio y quimioprospectivos, metabolómicos, farmacológicos, medicinales, etc. Todo 
lo anterior se suma a la razón de señalar que esta planta es una Asteraceae poco valorada pero que en la realidad posee una alta potencialidad química y biológica en el neotrópico.

\section{AGRADECIMIENTOS}

Producto derivado del proyecto INV-CIAS-1788 de la Vicerrectoría de Investigaciones de la UMNG - Vigencia 2015.

\section{REFERENCIAS}

1. Abad M, Bermejo P. 2007. Baccharis (Compositae): a review update. Arkivoc, 7:76-96.

2. Abad MJ, Bessa AL, Ballarin B, Aragón $O$, Gonzales E, Bermejo P. 2006. Anti-inflammatory activity of four Bolivian Baccharis species (Compositae). Journal of Ethnopharmacology, 103(3):338-344.

3. Abad MJ, Bessa AL, Bermejo P. 2005. Biologically active substances from the genus Baccharis L. (Compositae), In: Atta-ur-Rahman, Editor Studies in Natural Products Chemistry, Elsevier, 30:703-759.

4. Andersen OM, Jordheim M. 2006: The anthocyanins. In: Andersen OM, Markham KR (eds). Flavonoids chemistry, biochemistry and applications. CRC Press, Taylor and Francis, Boca Raton, FL, USA, 471-551.

5. Anzenbacher P, Zanger UM. 2012. Metabolism of drugs and other xenobiotics. Ed. John Wiley \& Sons, Weinheim, Germany, 543-550.

6. Arriaga-Giner FJ, Wollenweber E, Schober I, Dostal P, Braunt S. 1986. 2 $\beta$-hydroxyhautriwaic acid, a clerodane-type diterpenoid and other terpenoids from three Baccharis species. Phytochemistry, 25(3):719-721.

7. Bedascarrasbure, E. Maldonado, L. Álvarez, A. Rodríguez, E. 2004. Contenido de fenoles y flavonoides del propóleo argentino. Acta Farmacéutica Bonaerense, 23(3):369-372.

8. Biurrun F, Juliani RH, Lopez ML, Zygadlo JA. 2005. Essential Oil Composition of Baccharis tenella Hook. Et Arn. Journal of Essential Oil Research, 17(2), 122-123.

9. Bohlmann F, Kramp W, Jakupovic J, Robinson H, King RM. 1982. Diterpenes from Baccharis species. Phytochemistry, 21(2):399-403.

10 Brack EA. 1999. Diccionario enciclopédico de plantas útiles del Perú. Centro de Estudios Regionales Andinos Bartolomé de las Casas. Cuzco, Perú. 556 pp.

11. Bremer K. 1994. Asteraceae. Cladistics and classification. Timber Press. Portland, USA. 752 pp

12. Cabrera A, Crisci J, Delucchi G, Freire S, Giuliano D, Iharlegui L, Katinas L, Saenz A, Sancho G, Urtubey E. 2000. Catalogo Ilustrado de las Compuestas (=Aateraceae) de la Provincia De Buenos Aires. Argentina: Sistemática, Ecología y Usos. ProBiota, 1:1-137.

13. Callaway E, Cyranoski D. 2015. Anti-parasite drugs sweep Nobel prize in medicine 2015. Nature, 526:174-175.

14. Carraz M, Lavergne C, Jullian V, Wright M, Gairin JE, Gonzales de la Cruz M, Bourdy G. 2015. Antiproliferative activity and phenotypic modification induced by selected Peruvian medicinal plants on human hepatocellular carcinoma Hep3B cells. Journal of Ethnopharmacology, 166:185-199. 
15. Concha J, Gaston C, Hernández V. 2014. Essential oil composition of Baccharis linearis (Ruiz \& Pav.) and Baccharis paniculata DC. Leaves from Chile. American Journal of Essential Oils and Natural Products. 1(4):6-8.

16. Corrêa MP. 1984. Dicionário das plantas úteis do Brasil e das exóticas cultivadas. Imprensa Nacional - Rio de Janeiro. 1-6.

17. Cuatrecasas J. 1968. Notas adicionales, taxonómicas y corológicas, sobre Baccharis. Revista Academia Colombiana de Ciencias, 13(50):201- 226.

18. Daniel M. 2006. Medicinal Plants: Chemistry and Properties. Science Publishers, Enfield, $\mathrm{NH}$, EEUU, $300 \mathrm{pp}$.

19. Díaz-Piedrahita S, 1989. Origen y desarrollo de la sinanterologia en Colombia. Revista Academia Colombiana de Ciencias, 17:189-206.

20. Díaz-Piedrahita S, Rodríguez-Cabeza BV. 2012. Novedades en asteráceas colombianas - II. Revista Academia Colombiana de Ciencias, 36:501-515.

21. Freire S, Urtubey E, Giuliano D. 2007 Epidermal Characters of Baccharis (Asteraceae) Species used in Traditional Medicine. Caldasia, 29(1):23-38.

22. Funk VA, Susanna A, Stuessy T, Robinson H 2009. Classification of Compositae. Pp. 171189: In Systematics, Evolution, and Biogeography of Compositae (Funk VA, Susanna A, Stuessy T, Bayer R, eds). IAPT, Vienna, 965 pp.

23. Grecco S, Reimao J, Tempone A, Sartorelli P, Cunha R, Romoff P, Ferreira M, Favero O, Lago H. 2012. In vitro antileishmanial and antitrypanosomal activities of flavanones from Baccharis retusa DC. (Asteraceae). Experimental Parasitology, 130(2):141-145.
24. Guiliano D. 2001. Clasificación infragenérica de las especies Argentinas de Baccharis (Asteraceae, Astereae). Darwiniana, 39:131 -154.

25. Havsteen BH. 2002. The biochemistry and medical significance of the flavonoids. Pharmacology \& Therapeutics, 96(2-3):67-202.

26. Hoyos M, Yep M. 2008. Diseño de una formulación de aplicación tópica a base de Baccharis latifolia (Chilca), con efecto antiinflamatorio. Tesis para obtener título de Químico Farmacéutico. Universidad Nacional Mayor de San Marcos. Lima- Perú.

27. Jakupovic J, Schuster A, Ganzer U, Bohlmann F, Boldt PE. 1990. Sesqui- and diterpenes from Baccharis species. Phytochemistry, 29(7):2217-2222.

28. Jarvis BB, Mokhtari-Rejali N, Schenkel EP, Barros CS, Matzenbacher NI. 1991. Trichothecene mycotoxins from brazilian Baccharis species. Phytochemistry 30:789-797.

29. Jasinski V, Silva R, Pontarolo R, Budel J, Campos F. 2014. Morpho-anatomical characteristics of Baccharis glaziovii in support of its pharmacobotany. Revista Brasileira de Farmacognosia, 24(6):609-616.

30. Korbes CV. 1995. Manual de plantas medicinais. Francisco Beltrão: Associação de Estudos, 48ª Ed., Orientação e Assistência Rural, 188 pp.

31. Kurdelas R, Lima B, Tapia A, Feresin G, Gonzalez M, Rodríguez M, Zacchino S, Enriz R, Freile M. 2010. Antifungal activity of extracts and prenylated coumarins isolated from Baccharis darwinii Hook \& Arn. (Asteraceae). Molecules, 15:4898-4907.

32. Lago JHG, Romoff $P$, Fávero OA, Souza FO, Soares MG, Baraldi PT, Corrêa AG. 2008. 
Chemical composition of male and female Baccharis trimera (Less.) DC. (Asteraceae) essential oils. Biochemical Systematics and Ecology, 36(9):737-740.

33. Lonni A, Scarminio L, Silva L, Ferreira D. 2003. Differentiation of species of the Baccharis genus by HPLC and chemometric methods. Analitycal Sciences, 19:1013-1017.

34. Lonni A, Scarminio L, Silva L, Ferreira D. 2005. Numeral Taxonomy Characterization of Baccharis genus species by ultraviolet-visible spectrophotometry. Analitycal Sciences, 21:235- 239.

35. Malagarriga RP. 1976. Nomenclator Baccharidinarum omnium. Memoria de la Sociedad de Ciencias Naturales La Salle, 37:129-224.

36. Malizia RA, Cardell DA, Molli JS, González SB, Guerra PE, Grau RJ. 2005a. Volatile constituents of leaf oils from the genus Baccharis. Part I. B. racemosa (Ruiz et. Pav.) De Candolle and B. linearis (Ruiz et. Pav.) Persoon species from Argentina. Journal of Essential Oil Research, 17:103-106.

37. Malizia RA, Cardell DA, Molli JS, González SB, Guerra PE, Grau RJ. 2005b. Volatile constituents of leaf oils from the genus Baccharis. Part II. B. obovata Hooker et Arnott and B. salicifolia (Ruiz et. Pav.) Persoon species from Argentina. Journal of Essential Oil Research, 17:194-197.

38. Martínez S, Terraza E, Álvarez T, Manani O, Vila J, Mollinedo P. 2010. Actividad Antifúngica in Vitro de extractos polares de plantas del género Baccharis sobre fitopatogenos. Revista Boliviana de Química, 27(1):13-18.
39. Molares S, Gonzales B, Ladio A, Agueda M. 2009. Etnobotánica, anatomía y caracterización físico-química del aceite esencial de Baccharis obovata Hook. et Arn. (Asteraceae: Astereae). Acta Botánica Brasilera, 23(2):578-589.

40. Oliva R, Raimundo F, Valdés B. 2003. Especies ornamentales de la familia Asteraceae cultivadas en las áreas verdes de Sicilia occidental. Lagascalia, 23:75-84.

41. Paredes, B. 2002. Análisis y obtención de colorante natural a partir de la Baccharis latifolia (Chilca). Trabajo de tesis para obtener título de ingeniero textil. Universidad Técnica del Norte. Ibarra- Ecuador.

42. Parodi FJ, Fischer NH. 1988. Guaianolides from Baccharis salicina. Phytochemistry, 27(9):2987-2988.

43. Pérez-García F, Marín E, Adzet T, Cañigueral S. 2001. Activity of plant extracts on the respiratory burst and the stress protein synthesis. Phytomedicine, 8(1):31-38.

44. Pires P, Becker S. 2010. Actividade antimicrobiana dos extractos Baccharis dracunculifolia D.C (Asteraceae). Sabios- Revista de Saúde e Biologia, 5(2):1-6.

45. Raymond K. 2009. Flavonoids: biosynthesis, biological effects and dietary sources. Nova Science Publishers Inc., New York, 1-3 pp.

46. Reynel C, León J. 1990. Árboles y arbustos andinos para agroforestería y conservación de suelos, Lima, Perú, Proyecto FAO/Holanda/ DGFF, $361 \mathrm{p}$.

47. Sequeda-Castañeda LG, Célis C, LuengasCaicedo. 2015. Phytochemical and therapeutic 
use of Baccharis latifolia (Ruiz \& Pav.) Pers. (Asteraceae). PharmacologyOnLine, 2015(2):14-17.

48. Simões-Pires CA, Debenedetti S, Spegazzini E, Mentz LA, Matzenbacher NI, Limberger RP, Henrique AT. 2005. Investigation of the essential oil from eight species of Baccharis belonging to sect. Caulopterae (Asteraceae, Astereae): a taxonomic approach. Plant Systematics and Evolution, 253(1):23-32.

49. Singh A. 2006. Compendia of World's Medicinal Flora. CRC Press, Taylor and Francis Publishing Group. Boca Raton, FL, USA, 89-90 pp.

50. Smith N, Mori SA, Henderson A, Stevenson DWm, Heald SV (eds.). 2004. Flowering plants of the Neotropics. Princeton University Press. Princeton, USA. $694 \mathrm{pp}$

51. Soicke H, Leng-Peschlow E. 1987. Characterization of flavonoids from Baccharis trimera and their antihepatotoxic properties. Planta Medica, 53:37-39.

52. Tapia A, Rodríguez J, Theoduloz C, Lopez S, Egly G, Schameda G. 2004. Free radical scavengers and antioxidants from Baccharis grisebachii. Journal of Ethnopharmacology, 95(2):155-161.

53. Tapia JL. 2010. La familia Asteraceae. Desde el Herbario CICY, 2: 82-84.

54. Troiani H. 1985. Las especies de Baccharis (Compositae) de la provincial de la Pampa. Revista Facultad de Agronomía Universidad Nacional de la Pampa, 1:1-2.

55. Velásquez A. 2007. Actividad antimicrobiana de extractos de Franseria artemisioides, Rumex palistris, Baccharis latifolia, Cestrum parqui y Piper asterifolium frente a Staphylococcus aureus, Escherichia coli, Pseudomonas aeruginosa y Enterococcus faecalis. Universidad Mayor de San Andrés. Facultad de Ciencias Farmaceuticas y Bioquímicas. La paz-Bolivia.

56. Verdi G, Brighente C, Pizzolatti G. 2005. Género Baccharis (Asteraceae): aspectos químicos, económicos e biológicos. Quimica Nova, 28:85-94.

57. Wiersema J, León B. 2013. World Economic Plants. Second Edition. CRC Press., 341 pp.

58. Zapata B, Durán C, Stashenko E, Betancur-Galvis L, Mesa-Arango AC. 2010. Antifungal activity, cytotoxicity and composition of essential oils from the Asteraceae plant family. Revista Iberoamericana de Micología, 27(2):101-103.

59. Zdero C, Bohlmann F, Niemeyer HM. 1990. ent-Labdane glycosides from Baccharis pingraea. Phytochemistry, 29(8):2611-2616.

60. Zdero C, Bohlmann F, Solomon JC, King RM, Robinson H. 1989. ent-clerodanes and other constituents from bolivian Baccharis species. Phytochemistry, 28(2):531-542. 